\title{
Structure of Life Adjustments by Lung Cancer Patients with Decreased Performance Status
}

\author{
Naoko Horii ${ }^{1,2 *}$, Akiko Kondo ${ }^{3}$, Harumi Ejiri and Atsuko Maekawa ${ }^{4}$ \\ ${ }^{1}$ Nagoya University Graduate School of Medicine, Japan \\ ${ }^{2}$ Department of Nursing, College of Life and Health Sciences, Chubu University, Japan \\ ${ }^{3}$ School of Nursing, Graduate School of Nursing, Tokyo Women's Medical University, Japan \\ ${ }^{4}$ Department of Nursing, Nagoya University Graduate School of Medicine, Japan
}

\section{Abstract}

Purpose: The structural characteristics of life adjustments among lung cancer patients with decreased Performance Status (PS) were investigated.

Subjects and methods: Text analysis and cluster analysis were performed with data obtained from interviews of six lung cancer patients with PS 3 or 4 in one university hospital in Japan. The study was conducted from February to June 2009

Results: The mean age of subjects was 73.6 years. The text data included 623 records, and the number of keywords was 1,398. Keywords that appeared with high frequency were, in order, People (51), now (47), Disease (45), and Doctor (32). Positive type (173) and negative type (366) were extracted from a sensitivity analysis. Life adjustments of lung cancer patients were divided with a linguistic method into 20 categories: "Time period", "Other people", "Actions in daily life", "Symptoms", "Lung cancer", "Place of treatment", "Medical treatment", "Adjustment", "Death", "Physical condition", "Feelings of difficulty", "Relationships with others", "Enjoyment", "Doing one's best”, "Acceptance", "Living", "Attentiveness to others", "Belief", "Nursing care" and "Thankfulness." Four clusters were formed in the cluster analysis: Thoughts on receiving care, living on terms with one's physical condition, adjustments with awareness of death, and actions in daily life appropriate to the time.

Discussion: Life adjustments of hospitalized lung cancer patients with decreased PS are closely related to time period, treatment, symptoms and it is conjectured that patients make adjustments to their actions in daily life and thoughts with a positive attitude and feelings when they reach the state of sensing that they will not be cured.

Keywords: Lung cancer patient; Life adjustment; Text analysis

\section{Introduction}

Lung cancer has the highest prevalence rate and mortality among all cancers and the lung cancer prevalence rate is increasing worldwide [1]. In Japan lung cancer has been the leading cause of cancer deaths since 1998 [2]. At the time of diagnosis about 70\% of cases are in the advanced stage, so that expectations for a complete cure and prolongation of survival are rather poor. In recent years the concept of cancer survivorship has become pervasive in the field of cancer nursing. This includes the meaning of patients living in the way that suits them best from the time they are diagnosed with lung cancer until the end of life. In order to live in the way that suits them best while facing various daily issues from cancer, patients who have been told they have lung cancer need to make changes and adjustments in the way they have lived up to that time.

Studies in Japan and other countries related to the lives of lung cancer patients include studies on impediments in daily life $[3,4]$, mental state [5-7], and QOL [8,9]. In nearly all studies on adjustment to lung cancer [10-12], the focus is on psychosocial adjustment. However, few studies have comprehensively investigated how patients battling lung cancer make adjustments in their lives in terms of attitude and behaviors in attempting to continue living together with cancer.

In this study, life adjustment in lung cancer patients is defined as "adopting the best behaviors and frame of mind to live while facing the challenges experienced daily after developing lung cancer.”

The aim of this study was to elucidate the life adjustments of lung cancer patients who have reduced Performance Status (PS) due to progressing symptoms. For this purpose we conducted a text analysis to investigate the key words and structural characteristics that make up life adjustment. This will also serve as basic data for support of life adjustment in lung cancer patients.

\section{Methods}

\section{Subjects}

The subjects were six lung cancer patients hospitalized in the respiratory ward of one university hospital with about 1,000 beds in the Tokai region of Japan. These lung cancer patients had PS grade of 3 (more than $50 \%$ of the day spent lying in bed) or 4 (needed to be in bed all day).

\section{Data collection}

Data were collected using unstructured interviews. In the interviews, subjects were asked to speak freely on changes they had made in their lives after being diagnosed with lung cancer. The method of recruiting subjects was to have the ward charge nurse select patients who met the conditions. Then, after obtaining the permission of the attending physician, the researchers asked the patients to participate in the study.

*Corresponding author: Naoko Horii, Department of Nursing, College of Life and Health Sciences, Chubu University, 1200 Matsumoto-cho, Kasugai-city, Aichi-ken 487-8501, Japan, Tel: +81-568-51-5498; Fax: +81-568-51-5498; E-mail: n-horii@isc.chubu.ac.jp

Received September 01, 2012; Accepted October 25, 2012; Published October 30, 2012

Citation: Horii N, Kondo A, Ejiri H, Maekawa A (2012) Structure of Life Adjustments by Lung Cancer Patients with Decreased Performance Status. J Nurs Care S5:001. doi:10.4172/2167-1168.S5-001

Copyright: (C) 2012 Horii N, et al. This is an open-access article distributed unde the terms of the Creative Commons Attribution License, which permits unrestricted use, distribution, and reproduction in any medium, provided the original author and source are credited. 
The patient selection criteria, in addition to PS stage of 3 of 4 , were (1) having been informed of their lung cancer, (2) age of $\geq 20$ years, (3) ability to be interviewed for 20-30 min and (4) no serious psychiatric condition or disorder. The interviews were conducted in patient rooms (private rooms) with consideration for privacy and patients' conditions. The survey was conducted from February to April 2009.

\section{Analysis}

The text data obtained from the interviews were recorded with one meaningful sentence taken as one record. A text analysis was conducted using the IBM SPSS Text Analytics for Survey (TAfS) text analysis tool. In the analysis, (1) main keywords were extracted and (2) the main key words were categorized using the TAfS linguistic technique. In this method, key words that contain common terms are brought together in one category. Afterward, multiple researchers manually combined or deleted keywords repeatedly until agreement was reached. (3) Main keywords were categorized based on emotional type (positive or negative). (4) A network diagram was prepared and duplication between multiple categories (co-occurrence relation) was confirmed.

In addition, a cluster analysis was performed for the ultimately categorized data using SPSS Ver. 15 in order to confirm similarity. Between group average linkage was used to create clusters.

\section{Ethical considerations}

The study was approved by the ethics committees of the authors' university and the university hospital where the data were collected. In conducting the study the survey content was explained orally and in writing to the subjects. The survey results, including personal information, are stored and managed under strict rules so that they can be accessed only by the researchers, and anonymity was guaranteed in processing the results. Consent forms were obtained from patients who agreed to participate in the survey. Maximum concern was given for the

Table 1: Subject background.

\begin{tabular}{|c|c|c|c|}
\hline \multicolumn{2}{|c|}{ Attribute } & $\mathrm{n}$ & $(\mathrm{N}=6)$ \\
\hline \multirow{3}{*}{ Age } & Minimum & 66 & \\
\hline & Maximum & 77 & \\
\hline & Mean \pm SD & 73.6 & \pm 5.0 \\
\hline \multirow{2}{*}{ Sex } & Male & 3 & \\
\hline & Female & 3 & \\
\hline \multirow{2}{*}{ Histological type } & Adenocarcinoma & 5 & \\
\hline & Small-cell cancer & 1 & \\
\hline \multirow{4}{*}{ Stage } & 1 & 0 & \\
\hline & II & 1 & \\
\hline & III & 2 & \\
\hline & IV & 3 & \\
\hline \multirow{2}{*}{ PS } & 3 & 5 & \\
\hline & 4 & 1 & \\
\hline \multirow{5}{*}{$\begin{array}{c}\text { Main treatment } \\
\text { (multiple responses) }\end{array}$} & Underwent surgery & 2 & \\
\hline & Receiving chemotherapy & 4 & \\
\hline & Receiving radiotherapy & 0 & \\
\hline & Receiving oxygen therapy & 2 & \\
\hline & Receiving pain control & 3 & \\
\hline \multirow{2}{*}{ Employment status } & Employed & 0 & \\
\hline & Unemployed & 6 & \\
\hline \multirow{3}{*}{$\begin{array}{c}\text { No. of family members } \\
\text { in household }\end{array}$} & 1 & 0 & \\
\hline & 2 & 3 & \\
\hline & $\geq 3$ & 3 & \\
\hline
\end{tabular}

patients' mental and physical conditions in the timing of the interviews, with reference also to the opinions of the attending physicians.

\section{Results}

\section{Subject characteristics}

There were six subjects (three men, three women) with a mean age of 73.6 years (range $=66-76$ years). PS grade was 3 for five subjects and 4 for one subject (Table 1). The mean duration of disease since diagnosis was 38.8 months (range 8-96 months). The mean interview time was 40.2 minutes (range $=26-61$ minutes).

\section{Keyword extraction (word frequency analysis)}

The text data related to life adjustment consisted of 623 records. Data were divided into parts of speech and proper nouns. Things such as conjunctions, particles/auxiliary verbs, and punctuation that do not have meaning by themselves were excluded. As a result, a total of 1,398 words were extracted. In addition, multiple expressions that had the same meaning, such as disease, lung cancer, and cancer, were taken as synonyms and converted to the keyword, in this case "disease," that appeared the most frequently. Following the above preprocessing, 169 different keywords were ultimately extracted from the 1,398 words. The keywords that appeared with highest frequency were, in order, Now (94), Disease (86), Person (54), Go (50), Die (45), Hospital (44), Time (36), Doctor (35), Anticancer agent (31), Think (30), Leave the hospital (25), and Body (21) (Table 2).

\section{Categorization}

The 169 keywords were categorized, including categorization based on the linguistic method with a focus on similar concepts, and categorization based on emotional type (positive or negative). In the following, categories are indicated with quotation marks and the numerals in parentheses are frequency of appearance.

In the categorization based on the linguistic method, combination and exclusion of keywords was repeated manually while checking the actual raw data. Keywords thought to have similar meanings were then transformed to more comprehensive words and the categories were named. As a result, they were divided into 20 categories: "Time period" (189), "Other people" (179), "Actions in daily life" (177), "Symptoms" (124), "Lung cancer" (91), "Place of treatment" (87), "Medical treatment" (82), "Adjustment" (69), "Death" (58), "Physical condition" (49), "Feelings of difficulty" (49), "Relationships with others" (48), "Enjoyment" (42), "Doing one's best" (36), "Acceptance" (35), "Living" (34), "Attentiveness to others" (15), "Belief” (12), "Nursing care" (12), and "Thankfulness" (10).

In categorization based on emotional type, classification was based on keyword evaluation as positive $(\mathrm{P})$ or negative $(\mathrm{N})$. Classification was done into positive type (173), such as vitality (P-pleasurable), enjoyment (P-enjoyment), thanks to others (P-thankfulness), being happy (P-happiness), feeling good (P-general joy), and being all right (P-peace of mind), and negative types (366) such as hospitalization (N-poor physical condition), death (N-bad), unsavory (N-dissatisfaction), suffering (N-suffering), listlessness ( $\mathrm{N}$-discomfort), be worried ( $\mathrm{N}$-anxiety), and scared ( $\mathrm{N}$-fear).

\section{Network analysis (co-occurrence relation)}

The network diagram illustrates the relationship between linguistic expressions with strong links. Larger circles show words that appeared with greater frequency. Co-occurrence relation is the probability that a given word will appear in the same sentence with another word. The 
Table 2: Categories and main key words.

\begin{tabular}{|c|c|c|c|c|c|}
\hline Category & $\begin{array}{c}\text { Key word } \\
\text { (in order of higher frequency) }\end{array}$ & App. freq. & Category & $\begin{array}{c}\text { Key word } \\
\text { (in order of higher frequency) }\end{array}$ & App. freq. \\
\hline \multirow{4}{*}{ Time period } & Now & 94 & \multirow{2}{*}{ Death } & Death & 45 \\
\hline & Time & 36 & & Final days & 10 \\
\hline & Past & 34 & Physical condition & Physical condition & 13 \\
\hline & Leaving hospital & 25 & rilysteal cunturtion & Strength & 13 \\
\hline \multirow{4}{*}{ Other people } & People & 54 & \multirow{2}{*}{ Feelings of difficulty } & Scared & 19 \\
\hline & Father & 31 & & Dislike & 6 \\
\hline & Friends & 14 & \multirow{3}{*}{ Relationships with others } & Talk & 18 \\
\hline & Visitors in hospital & 12 & & Listen & 16 \\
\hline \multirow{5}{*}{ Actions in daily life } & Go & 50 & & Spend time with & 6 \\
\hline & Meal & 20 & \multirow{2}{*}{ Enjoyment } & Travel & 16 \\
\hline & Work & 19 & & Exercise & 14 \\
\hline & Go out & 14 & \multirow{2}{*}{ Do one's best } & Do my best & 11 \\
\hline & Sleep & 12 & & Strong & 7 \\
\hline \multirow{4}{*}{ Symptoms } & Body & 21 & \multirow{2}{*}{ Acceptance } & Acceptance & 17 \\
\hline & Pain & 17 & & Recognition & 8 \\
\hline & Strange & 8 & \multirow{2}{*}{ Living } & Living & 11 \\
\hline & Listless & 8 & & Vitality & 7 \\
\hline \multirow{2}{*}{ Lung cancer } & Disease & 86 & \multirow{2}{*}{ Attentiveness to others } & Vital energy & 9 \\
\hline & Metastasis & 5 & & Inconvenience to others & 6 \\
\hline \multirow{3}{*}{ Place of treatment } & Hospital & 44 & \multirow{2}{*}{ Belief } & Protect & 9 \\
\hline & Home & 24 & & Belief & 3 \\
\hline & Hospice & 6 & \multirow{2}{*}{ Nursing care } & Nursing care & 5 \\
\hline \multirow{3}{*}{ Medical treatment } & Doctor & 35 & & Services & 4 \\
\hline & Anticancer agent & 31 & \multirow{2}{*}{ Thankfulness } & Thanks & 4 \\
\hline & Surgery & 18 & & Thanks to others & 3 \\
\hline \multirow{3}{*}{ Adjustment } & Think & 30 & & & \\
\hline & Being careful & 9 & & & \\
\hline & Adjustment & 6 & & & \\
\hline
\end{tabular}

line depicting co-occurrence goes from broken lines to thick solid lines as the co-occurrence becomes stronger. In the present study a network diagram is drawn with "Adjustment" as the central word to confirm the co-occurrence relation of the 20 category names (Figure 1). The numerals in brackets in the following express the appearance rate of other categories when "Adjustment" is taken to be $100 \%$.

Co-occurrence relations with "Adjustment" were seen in 17 categories, including "Time period" (39.1), "Actions in daily life" (26.1), "Symptoms" (23.2), "Other people" (18.8), "Lung cancer" (17.4), "Place of treatment" (14.5), "Feelings of difficulty" (14.5), "Medical treatment" (14.5), and "Death" (13.0). Absolutely no co-occurrence relation with "Adjustment" was seen for the two categories of "Thankfulness" and "Attentiveness to others."

\section{Cluster formation}

A dendrogram was created for the 20 categories by calculating the squared Euclidean distance (Figure 2). After confirming similarity, the characteristics of each cluster were dissected with the 20 identified Euclidean distances, and four clusters were formed. The clusters were named by the researchers. Attempts were made to use the content of each item as fully as possible in the naming, while considering the analysis results. The first cluster was named Thoughts on receiving care, with the categories of "Care", "Thankfulness", "Belief", and "Attentiveness to others." The second cluster was named Coming to terms with one's physical condition, with categories including "Living", "Acceptance", "Physical condition" and "Feelings of difficulty." The third cluster was named Adjustments with awareness of death, with categories including "Death", "Adjustment", "Place of care", and "Lung cancer." The fourth cluster was named Actions in daily life appropriate to the time, with the categories of "Symptoms", "Actions in daily life", "Time period" and "Other people."

\section{Discussion}

In this study, interviews were conducted to understand the life adjustments confronted by lung cancer patients with reduced PS. From the interviews, 169 different key words were extracted and grouped in 20 categories. From a word frequency analysis, time period was thought to be an important key word in the life adjustment of lung cancer patients, from the words/phrases Now, Time, and Leave the hospital that occurred with the greatest frequency. It may be inferred from words such as Disease, Hospital, Doctor, and Anticancer agent that medical treatment influences life adjustment.

From the results of sensitivity analysis, life adjustments in lung cancer patients with decreased PS may be assessed. More negative key words, such as Hospitalization, Death, Unsavory, and Suffering were identified than positive key words such as Vitality, Enjoyment, Being happy, and Feeling good. It may be said that while lung cancer patients simultaneously hold both positive and negative assessments of life adjustment, the overall assessment is negative.

Four clusters were formed through confirmation of similarity with cluster analysis: Thoughts on receiving care, coming to terms with one's physical condition, Adjustments with awareness of death, and Actions in daily life appropriate to the time.

In thoughts on receiving care, an attitude is seen in which patients accept care and are thankful, while being careful of their surroundings because of inability to do things themselves that come with decreased PS, and holding their own beliefs close. This can be said to be emotional 


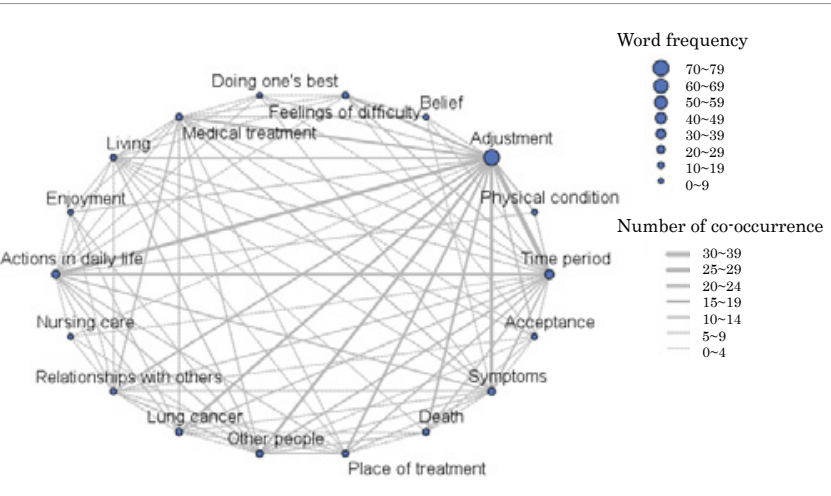

Figure 1: Network diagram.

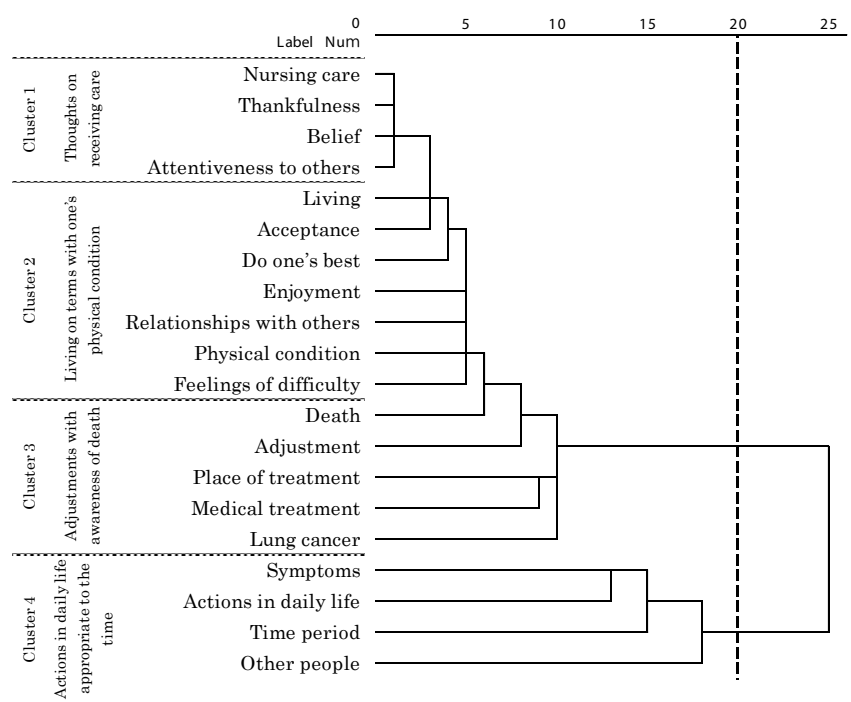

Figure 2: Clusters' dendrogram.

adjustments in people who spend more than half of the day lying down and accepting care.

In coming to terms with one's physical condition, there is also acceptance in which patients are aware of their poor physical condition and have feelings of difficulty, and show an attitude of making effort while treasuring pleasure and social relationships. Kissane [13] stated that while a certain level of suffering itself is seen in the influence of physical symptoms on individual patients, it should be noted that the severity of unrelieved symptoms is not necessarily related directly to suffering. O'Brien [14] wrote that a positive attitude in advanced cancer patients affects emotional adaptation. Thus it is considered that, even with poor physical condition, support from enjoyment and social relationships is tied to coming to terms with one's physical condition.

Adjustments with awareness of death is a patient attitude of making adjustments for one's limited remaining time, such as where to spend one's final days, and who to spend one's final time with. This adjustment is made when a person senses that death cannot be avoided.

Actions in daily life appropriate to the time are an attitude in which patients adjust their actions in daily life to avoid being a physical and mental burden on others during treatment and with the appearance of symptoms. It has been shown clinically that patients experience multiple symptoms accompanying the progression of cancer $[15,16]$. As unavoidable adverse effects from treatment occur and symptoms appear, patients make adjustments to the way they live so as not to be a physical or mental burden.

Overall, life adjustments in lung cancer patients are closely related to the time period, treatment, and symptoms, and it is conjectured that people make adjustments to their actions in daily life and thoughts with a positive attitude in situations where they cannot but help that sense that they will not be cured.

From the above, periods of change are key points in nursing interventions for life adjustments in lung cancer patients with decreased PS. As a consequence of the change in patients that takes them in a single direction with death in mind, medical personnel need to consider the timing and method of communicating bad news to patients and their families. Patients are aware of changes in themselves with the progression of symptoms or treatment, which leads to a mental state in which they ask themselves how to deal with their disease and choose a way to live. Support to help patients find appositive meaning in their current lives is also needed. Lazarus stated that a positive reassessment of one's circumstances can not only change stressful situations but also regulate emotions [17]. If patients can change their interpretation of lung cancer and attach a positive meaning to it, they may be able to control the emotions that arise from experiencing daily life with lung cancer. This may help them to accept their present circumstances, leading to positive life adjustments.

\section{Future Issues}

In analyzing the subjective experiences of life adjustments of lung cancer patients, maintaining objectivity is a major issue. In this study, the text analysis conducted using a computer was meaningful in that it helped to ensure objectivity. However, there are limits to the conceptual analysis since the text analysis is based on words separated from context, which is an important factor in the formation of subjective experience. In addition, the analysis results are based on data obtained from a group of 6 Japanese subjects who survived lung cancer for 39 months on average. Because the sample size was small and most of the study patients survived beyond the expected life span for lung cancer patients, generalisability of the study is limited. In the future it will be necessary to increase the numbers of facilities and subjects while also creating coding rules and deepening the analysis artificially.

\section{Acknowledgments}

The authors express their deep appreciation to all the participants, who graciously cooperated in this study despite their ongoing treatment, and to everyone at the participating hospitals for their tremendous cooperation.

\section{References}

1. Parkin DM (2001) Global cancer statistics in the year 2000. Lancet Oncol 2 533-543.

2. Health and Global Policy Institute (2011) Cancer patient attitude survey 2010.

3. Minagawa T, Kawasaki K, Noto Y, Amanai Y, Yamauchi H, et al. (2004) A study on quality of life issues of lung cancer survivors. Bull Health Sci Hirosaki 3: 1-7.

4. Prasertsri N, Holden J, Keefe FJ, Wilkie DJ (2011) Repressive coping style: relationships with depression, pain, and pain coping strategies in lung cancer outpatients. Lung Cancer 71: 235-240.

5. Faller H, Schmidt M (2004) Prognostic value of depressive coping and depression in survival of lung cancer patients. Psychooncology 13: 359-363.

6. Nakaya N, Saito-nakaya K, Akechi T, Kuriyama S, Inagaki M, et al. (2008) Negative psychological aspects and survival in lung cancer patients. Psychooncology 17: 466-473.

7. Berendes D, Keefe FJ, Somers TJ, Kothadia SM, Porter LS, et al. (2010) Hope in the context of lung cancer: relationships of hope to symptoms and psychological distress. J Pain Symptom Manage 40: 174-182. 
Citation: Horii N, Kondo A, Ejiri H, Maekawa A (2012) Structure of Life Adjustments by Lung Cancer Patients with Decreased Performance Status. J Nurs Care S5:001. doi:10.4172/2167-1168.S5-001

8. Kawasaki Y, Kosyobu T, Watanabe E, Okazaki R, Toge H, et al. (2007) Examination of factors in lung cancer patients' QOL. Shimane J Med Sci 27: 50-56.

9. John LD (2010) Self-care strategies used by patients with lung cancer to promote quality of life. Oncol Nurs Forum 37: 339-347.

10. Klemm PR (1994) Variables influencing psychosocial adjustment in lung cancer: a preliminary study. Oncol Nurs Forum 21: 1059-1062.

11. Badr H, Taylor CL (2008) Effects of relationship maintenance on psychological distress and dyadic adjustment among couples coping with lung cancer. Health Psychol 27: 616-627.

12. Butler L, Downe-Wamboldt B, Melanson P, Coulter L, Keefe J, et al. (2006) Prevalence, correlates, and costs of patients with poor adjustment to mixed cancers. Cancer Nurs 29: 9-16.
13. Kissane DW (2000) Psychospiritual and existential distress. The challenge for palliative care. Aust Fam Physician 29: 1022-1025.

14. O'Brien CW, Moorey S (2010) Outlook and adaptation in advanced cancer: a systematic review. Psychooncology 19: 1239-1249.

15. Dodd MJ, Miaskowski C, Paul SM (2001) Symptom clusters and their effect on the functional status of patients with cancer. Oncol Nurs Forum 28: 465470.

16. Esper P, Heidrich D (2005) Symptom clusters in advanced illness. Semin Oncol Nurs 21: 20-28.

17. Lazarus RS (1999) Stress and Emotion: A New Synthesis. Free Association Books, London.
This article was originally published in a special issue, Professional Issues and Trends in Nursing in Japan handled by Editor(s). Dr. Akiko Kondo, Tokyo Women's Medical University, Japan 